ISSN 0103-9954

\title{
UTILIZAÇÃO DA MADEIRA DE CANAFÍSTULA (Peltophorum dubium (Spreng.) Taub.) NA CONFECÇÃO DE CHAPAS DE MADEIRA AGLOMERADAS
}

\author{
USE OF CANAFÍSTULA WOOD (Peltophorum dubium (Spreng.) Taub.) IN THE \\ MANUFACTURE OF PARTICLEBOARDS
}

Karina Soares Modes ${ }^{1}$ Magnos Alan Vivian ${ }^{2}$ Daniela Silva Lilge ${ }^{3}$

Rafael Rodolfo de Melo ${ }^{4}$ Elio José Santini ${ }^{5}$ Clovis Roberto Haselein ${ }^{5}$

\begin{abstract}
RESUMO
A presente pesquisa objetivou avaliar preliminarmente o potencial de uso da madeira de canafístula (Peltophorum dubium (Spreng.) Taub.), para produção de chapas de partículas aglomeradas, coladas com adesivos à base de tanino e ureia-formaldeído, utilizados numa proporção de $9 \%$ com base no peso seco das partículas. Para cada tratamento, foram confeccionadas três chapas com densidade nominal de 0,63 $\mathrm{g} / \mathrm{cm}^{3}$ de onde se extraíram corpos de prova para verificação das propriedades físicas de absorção d'água em peso e volume e inchamento em espessura por 2 e 24 horas de imersão em água, e as propriedades mecânicas de flexão estática (MOE e MOR), arrancamento de parafusos (RAP) e ligação interna (LI), segundo dimensões e procedimentos prescritos pela norma ASTM D-1037. Os resultados dos ensaios físico-mecânicos foram comparados com o padrão estabelecido pelas normas ANSI A208.1-1987 e DIN 68761 (1)-1961, (3)-1971. De uma maneira geral, o uso de adesivo à base de ureia resultou em chapas com tendência a maior absorção d'água, ao passo que os valores de inchamento em espessura para ambos os tratamentos, se mantiveram de acordo com a norma. Com exceção do MOR, os demais valores de resistência dos painéis confeccionados com adesivo à base de ureia-formaldeído ficaram abaixo do mínimo exigido pela norma. Para o adesivo à base de tanino, todas as propriedades foram superiores, com exceção da RAP. Esses resultados evidenciam que a madeira de canafístula, quando agregada com adesivo à base de tanino-formaldeído, pode ser uma fonte alternativa de matéria-prima para a produção de chapas aglomeradas.
\end{abstract}

Palavras-chave: Peltophorum dubium; chapas aglomeradas; tanino-formaldeído; ureia-formaldeído.

\begin{abstract}
This study was carried out to evaluate the potential of use of canafístula wood (Peltophorum dubium (Spreng.) Taub.) in manufacturing particleboard, bonded with tannin and urea-formaldehyde adhesives used in a ratio of $9 \%$ of the particles oven-dry weight. For each treatment, three boards were made with a nominal density of $0.63 \mathrm{~g} / \mathrm{cm}^{3}$. The properties evaluated were the water absorption for two and 24 hours of immersion and the thickness swelling, the static bending (MOE and MOR), the screw withdrawal (RAP) and the internal bonding (LI), according to procedures prescribed by ASTM
\end{abstract}

1. Engenheira Florestal, MSc., Professora Assistente do Departamento de Engenharia Florestal, Universidade Federal de Rondônia, Campus Rolim de Moura, Av. Norte-Sul, 7300, CEP 78987-000, Rolim de Moura (RO). ksmodes@gmail.com

2. Engenheiro Florestal, Doutorando do Programa de Pós-graduação em Recursos Florestais, Departamento de Ciências Florestais, Escola Superior de Agricultura "Luiz de Queiroz" - ESALQ/USP, Av. Pádua Dias 11, CEP 13418-900, Piracicaba (SP). magnosalan@yahoo.com.br

3. Engenheira Florestal, MSc., Professora Assistente do Departamento de Engenharia Florestal, Universidade Federal do Pampa, Campus São Gabriel, Av. Antônio Trilha, 1847, CEP 97300-000, São Gabriel (RS). danielalilge@hotmail.com

4. Engenheiro Florestal, MSc., Professor Assistente da Universidade Federal do Piauí, Campus Professora Cinobelina Elvas, BR 135, Km 03, CEP 64900-000, Bom Jesus (PI).rrmelo2@yahoo.com.br

5. Engenheiro Florestal, Dr., Professor Associado do Departamento de Ciências Florestais, Centro de Ciências Rurais, Universidade Federal de Santa Maria, Av. Roraima, 1000, CEP 97105-900, Santa Maria (RS). ejsantini@gmail.com, clovis.haselein@ufsm.br

Recebido para publicação em 26/05/2009 e aceito em 20/12/2010

Ci. F1., v. 22, n. 1, jan.-mar., 2012 
D-1037 (2000). The results were compared with standards established by ANSI A208.1 (1987) and DIN 68761 (1971). The urea-formaldehyde adhesive increased the water absorption of boards and the data results of thickness swelling tests were in accordance with the standards for both treatments. With the exception of the MOR, the resistance data results from the urea bonded boards were below of the minimum standard values; for tannin, all the properties were superior, with the exception of the RAP. According to the results of this study, canafístula wood, when combined with tannin-formaldehyde adhesive, can be an alternative source of raw material for particleboard production.

Keywords: Peltophorum dubium; particleboard; tannin formaldehyde; urea formaldehyde.

\section{INTRODUÇÃO}

Conforme Azambuja et al., (2006), atualmente a quase totalidade da madeira utilizada na produção de painéis aglomerados, no Brasil, é proveniente de florestas plantadas, que é também utilizada para produção de polpa de papel, madeira serrada e beneficiada, lâminas para painéis compensados e, mais recentemente, para produção de painéis de fibras de média densidade (MDF).

De acordo com Brito et al. (2004), as chapas de partículas vêm apresentando as maiores taxas de crescimento de produção, dentre os produtos à base de madeira, em razão da diversidade de opções disponíveis e da flexibilidade de aplicação para os mais diversos fins. Deve-se considerar também que o desempenho destes materiais em serviço vai ser dependente dos processos utilizados na sua manufatura e do tipo de matéria-prima empregada e sua geometria, bem como da interação desta última com os aditivos e adesivos empregados, que também conferem uma ampla variação nas propriedades do produto acabado.

As indústrias de painéis aglomerados no Brasil utilizam preferencialmente cavacos de madeira de reflorestamentos, provenientes de pinus e de algumas espécies de eucalipto, o que determina inclusive uma melhor qualidade do produto, tendo em vista o maior controle da homogeneidade da matéria-prima (CARASCHI et al., 2009).

Portanto, diante da demanda crescente pela madeira de reflorestamento na constituição dos mais diversos tipos de chapas de madeira, combinada com a necessidade de suprimento cada vez maior por esses tipos de produtos, surge também a necessidade de emprego específico da madeira de espécies alternativas, assim como o aproveitamento de resíduos destas mesmas espécies.

Segundo Nahuz (2001), para florestas nativas, em termos práticos, isto significa aperfeiçoar o seu uso, maximizando a contribuição das florestas já em exploração, por meio da ampliação do número de espécies utilizadas, como também, pela possibilidade de redução do volume de resíduos acumulados na exploração e no processamento das mesmas.

A indústria da madeira vista de maneira global, usa os recursos naturais de maneira ineficiente, tanto na obtenção da matéria-prima, quanto na fase de processamento dos produtos e no descarte dos mesmos no fim de sua vida útil, significando uma grande exploração dos recursos madeireiros, e a grande geração de resíduos é a prova desta ineficiência (TEIXEIRA e CÉSAR, 2006).

O uso da madeira de pinus na confecção de chapas aglomeradas foi abordagem de um vasto número de pesquisas, apenas citando como exemplo os trabalhos de Dacosta et al. (2005a,b), Haselein et al. (2002), Hillig et al. (2004), Iwakiri et al. (2005), Iwakiri et al. (2001), Brito et al. (2006) e Vital et al. (2004). Da mesma forma, tão vasto quanto, é o uso da madeira de Eucalyptus, em que se encaixam os trabalhos de Gonçalves et al. (2003), Maciel et al. (2004), Iwakiri et al. (2000) e Tostes et al. (2004), entre tantos outros.

Apossibilidadedeusoda madeira de espécies alternativas e outros produtos lignocelulósicos na confecção de chapas aglomeradas está sendo cada vez mais considerada, e já é possível observar a iniciativa de um incipiente número de pesquisadores trabalhando nesta questão. Para Vidaurre et al. (2004), a disponibilidade de estudos sobre o emprego de espécies nativas para usos múltiplos é praticamente inexpressiva, sendo fatores limitantes o desconhecimento sobre os aspectos silviculturais e tecnológicos das referidas espécies verificando-se, portanto, um verdadeiro descompasso ao se considerar que, atualmente, é incontestável a importância do descobrimento de matérias-primas alternativas. Nesse contexto, 
a escolha da espécie é uma das variáveis mais importantes no processo de fabricação de chapas aglomeradas, pois dependendo das características do material, pode fornecer chapas de madeira pura ou misturada à outra, resultando num painel com características diferenciadas e, em último caso, como agente de enchimento.

Iwakiri et al. (2004) observaram que a madeira de Grevillea robusta, pode ser uma fonte alternativa de matéria-prima para a confecção de produtos com densidades de $0,8 \mathrm{~g} / \mathrm{cm}^{3}$, um valor pouco superior àquele de painéis comerciais, usualmente produzidos com densidade na faixa de 0,65 a $0,70 \mathrm{~g} / \mathrm{cm}^{3}$. Nunes et al. (1999), ao empregar o estipe de Euterpe edulis (palmiteiro), obtiveram bons resultados em relação à ligação interna e ao inchamento em espessura, porém com alguns valores de módulo elasticidade e de ruptura inferiores ao mínimo estipulado pela norma empregada. Azambuja et al. (2006), avaliando o uso da madeira de Manilkara huberi ducke (maçaranduba) na confecção de chapas de densidade $0,75 \mathrm{~g} / \mathrm{cm}^{3}$, encontraram valores satisfatórios de carga em relação ao módulo de ruptura, e superiores na maioria dos corpos de prova testados para a ligação interna, porém módulos de elasticidades inferiores ao mínimo exigido pela norma utilizada.

As madeiras de Anadenantheramacrocarpa (angico), Prosopis juliflora (algaroba) e Mimosa tenuiflora (jurema-preta), foram empregadas por Nascimento e Lahr (2003) na manufatura de painéis aglomerados. Os autores verificaram que as chapas produzidas com essas três espécies apresentaram valores de resistência mecânica à flexão estática, tração paralela e perpendicular às faces, resistência ao arrancamento de parafusos e características de inchamento e absorção de água mais favoráveis em relação às obtidas para as chapas industrializadas que usam como matériaprima partículas de madeira dos gêneros Pinus e Eucalyptus. Vidaurre et al. (2004) ao utilizarem as madeiras de Schizolobium parahyba (guapuruvu) e Erytrina verna (mulungu) na confecção de painéis OSB de densidade nominal $0,65 \mathrm{~g} / \mathrm{cm}^{3}$ constataram o elevado potencial de uso das mesmas.

Esses estudos mostram que as chapas de madeira reconstituídas produzidas com espécies alternativas, apresentam propriedades tão satisfatórias quanto às observadas em chapas confeccionadas com madeiras de florestas plantadas de maior tradição, evidenciando a possibilidade de sua utilização em nível industrial.

Nesse sentido, a busca de informações sobre espécies alternativas de rápido crescimento e propriedades tecnológicas compatíveis com a utilização dos diversos tipos de chapas aglomeradas inclui a canafístula (Peltophorum dubium) entre as consideradas promissoras. De acordo com o IPT (1978), a massa específica da madeira de árvores adultas está em torno de $0,80 \mathrm{~g} / \mathrm{cm}^{3}$, mas para madeira de povoamentos jovens, Vivian et al. (2008), encontraram uma massa específica básica média de $0,5 \mathrm{~g} / \mathrm{cm}^{3}$. Esse valor se enquadra dentro da faixa de 0,4 a $0,6 \mathrm{~g} / \mathrm{cm}^{3}$ mencionada por Hillig (2000) como apropriada para madeiras utilizadas na confecção de chapas.

É considerada uma espécie importante para programas de florestamento e reflorestamento em razão do comprovado valor econômico da madeira e do aceitável desempenho silvicultural (INOUE et al., 1984). Essas características de crescimento aliadas às boas propriedades tecnológicas da madeira justificam a realização de estudos sobre a viabilidade do emprego da madeira de povoamentos jovens de canafístula como matéria-prima alternativa para usos múltiplos, incluindo a confecção de painéis de madeira reconstituída.

Assim, visando à ampliação do conhecimento sobre o aproveitamento de essências nativas de rápido crescimento $\mathrm{e}$ a inserção da espécie entre as fornecedoras de matéria-prima para a indústria de chapas de madeira reconstituída, realizou-se este estudo com o objetivo de avaliar o potencial tecnológico da madeira de canafístula (Peltophorum dubium (Spreng.) Taub.) para a fabricação de chapas aglomeradas, utilizando adesivos à base de tanino e de ureiaformaldeído.

\section{MATERIAL E MÉTODO}

\section{Caracterização do material utilizado na confecção das chapas}

As partículas utilizadas nesse estudo foram obtidas de toras de Peltophorum dubium (Spreng.) Taub.), retiradas entre as posições 0,3 e $1,30 \mathrm{~m}$ da altura comercial de 6 árvores com, aproximadamente, 10 anos de idade, obtidas em um povoamento da Fundação Estadual de Pesquisa Agropecuária - FEPAGRO FLORESTAS, no município de Santa Maria-RS.

As partículas resultantes, obtidas por meio de uma plaina desengrossadeira, com dimensões 
médias de $0,8 \mathrm{~mm}$ de espessura, $20 \mathrm{~mm}$ de comprimento e largura variável, passaram por peneira para eliminação dos finos e permaneceram em estufa a $60^{\circ} \mathrm{C}$ até aproximadamente $5 \%$ de umidade, antes da confecção das chapas (Figura 1).

Como agentes aglutinantes foram empregados adesivos à base de tanino e de ureia, ambos numa proporção de $9 \%$, calculado com base no peso seco das partículas de madeira. O adesivo de tanino foi produzido em laboratório, a partir do tanino em pó extraído de acácia-negra (Acacia mearnsii De Wild.), o qual foi diluído a $50 \%$ em água, onde permaneceu por 24 horas para atingir a total hidratação. Para sua formulação, empregouse uma solução de 100 partes de sólidos de tanino (solução 50\%) para 10 partes de sólidos de formalina (solução 37\%), a qual foi adicionada apenas no momento da aplicação do adesivo. A solução teve seu pH ajustado para 8. Como adesivo de ureia, foi empregada uma resina produzida industrialmente, contendo aproximadamente $65 \%$ de sólidos.

Para ambos os tratamentos, seguindo a aplicação do adesivo, adicionou-se $1 \%$ de parafina (emulsão 50\%), com base no peso seco das partículas.

\section{Produção das chapas}

O experimento consistiu de dois tratamentos, estabelecidos conforme o tipo de adesivo utilizado: tanino-formaldeído e ureia-formaldeído. A composição dos painéis foi pré-estabelecida de modo que apresentassem $90 \%$ de partículas, 9\% de adesivo e $1 \%$ de parafina, e massa específica de 0,63 $\mathrm{g} / \mathrm{cm}^{3}$, ao teor de umidade de equilíbrio de $8 \%$. A pré-determinação da massa específica foi baseada no peso seco das partículas e no teor de sólidos do adesivo e da parafina utilizados.

As partículas de madeira, depois de secas até o teor de umidade requerido, foram introduzidas em um tambor rotativo, onde se procedeu a sua homogeneização com o adesivo e parafina, aplicados por meio de pistola acionada por compressor de ar, com pressão de $8 \mathrm{kgf} / \mathrm{cm}^{2}$ e vazão de $50 \mathrm{~g} /$ minuto. Seguida da aplicação do adesivo, e anteriormente à adição da parafina, foi aplicada a água necessária para ajustar o teor de umidade do colchão a aproximadamente $12 \%$ para ureia-formaldeído e $20 \%$ para tanino-formaldeído, conforme as recomendações de Hillig (2000), visando adquirir chapas com melhores propriedades físico-mecânicas.

Posteriormente, esta mistura gerada foi submetida à pesagem e dispersa sobre uma chapa de aço galvanizado no interior de um molde de madeira, com dimensões preconizadas para a chapa, para então, ser submetida à prensagem manual a temperatura ambiente por aproximadamente 2 minutos.

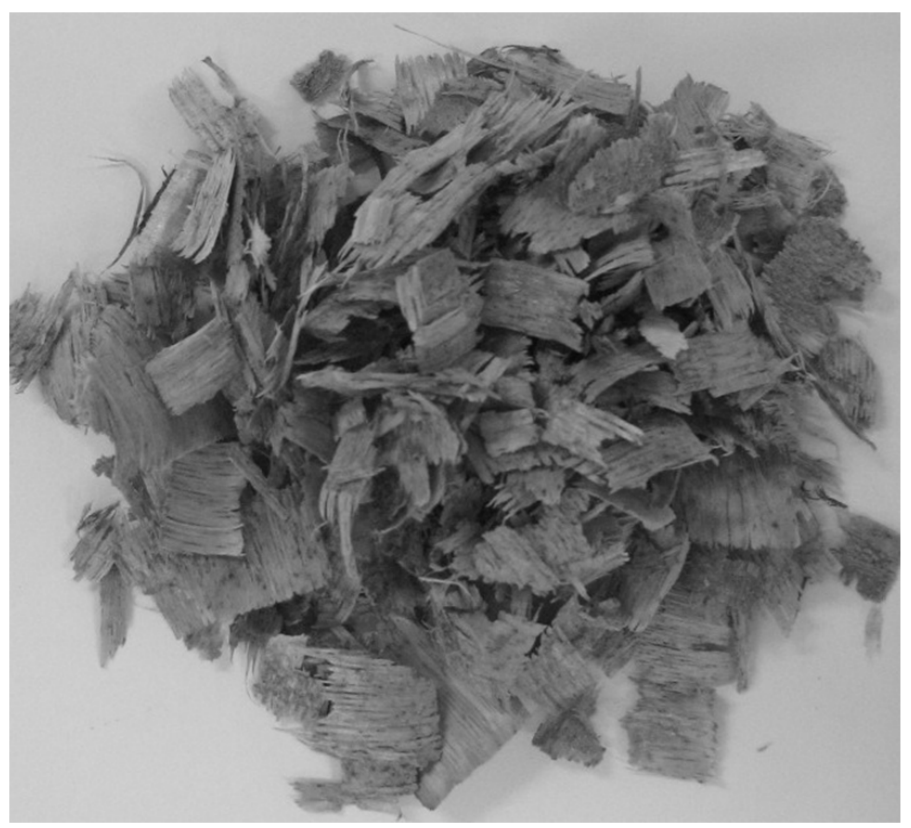

FIGURA 1: Partículas da madeira de canafístula (Peltophorum dubium) utilizadas na confecção das chapas.

FIGURE 1: Wood particles of canafístula wood (Peltophorum dubium) used to manufacture the particleboard. 
A prensagem definitiva foi conduzida em prensa hidráulica sob pressão específica de $30 \mathrm{kgf} /$ $\mathrm{cm}^{2}$, e a temperatura dos pratos ajustada para $180^{\circ} \mathrm{C}$, por tempos de prensagem de 12 e 8 minutos, para os painéis à base de tanino e ureia-formaldeído, respectivamente.

Confeccionaram-se três chapas para cada tratamento, com dimensões nominais de $50 \mathrm{~cm}$ de aresta e $0,95 \mathrm{~cm}$ de espessura nominal, esta última ajustada por meio de duas barras de aço colocadas dos dois lados do painel, limitando o fechamento da prensa. Depois de prensadas e aparadas as bordas em serra circular, as chapas foram levadas à câmara climatizada a $20^{\circ} \mathrm{C}$ e $65 \%$ de umidade relativa até atingirem o equilíbrio a $12 \%$ de umidade para posterior retirada de corpos de prova destinados aos ensaios.

\section{Avaliação das chapas \\ Testes fisico-mecânicos}

Os testes físico-mecânicos foram realizados segundo a norma ASTM D1037-1995 e os resultados interpretados com o auxílio do pacote estatístico ASSISTAT, utilizando o teste T, e comparados aos padrões de qualidade exigidos pelas normas ANSI A 208.1-1987 e DIN 68761 (1)-1961, (3)-1971.

As chapas foram avaliadas por meio dos testes de absorção d'água em peso (AP), absorção d'água em volume (AV), inchamento em espessura (IE), flexão estática (MOE e MOR), arrancamento de parafuso (RAP) e ligação interna (LI).

Para avaliação da propriedade de absorção d'água em peso e volume, e do inchamento em espessura dos painéis, confeccionaram-se corpos de prova de $150 \times 150 \mathrm{~mm}$ e destes, foram tomadas a espessura média utilizando um relógio adaptado para tal finalidade, a largura e comprimento com paquímetro e o peso em balança analítica eletrônica. Este procedimento foi realizado nos corpos de prova depois de condicionados a $20^{\circ} \mathrm{C}$ e $65 \%$ de umidade relativa, e repetido após 2 e 24 horas de imersão dos mesmos em água. Assim, com base nos dados registrados na primeira tomada de dados, e daqueles das peças depois de imersas, foram obtidas as seguintes variáveis: AP2h (absorção d'água em peso 2 horas) e AP24h (absorção d'água em peso 24 horas); AV2h (absorção d'água em volume 2 horas) e AV24h (absorção d'água em volume 24 horas), conforme Hillig et al., (2004); IE2h (inchamento em espessura 2 horas) e IE24h (inchamento em espessura 24 horas). Os cálculos destas variáveis, para cada tratamento, são apresentados nas Equações 1, 2 e 3.
Após todas as avaliações, procedeu-se a secagem dos corpos de prova em estufa a $103^{\circ} \mathrm{C}$ para determinação do TUe (teor de umidade de equilíbrio à condição de $20^{\circ} \mathrm{C}$ e $65 \%$ UR), TU2h (teor de umidade após 2 horas de imersão em água) e TU24h (teor de umidade após 24 horas de imersão em água).

$$
\begin{aligned}
& A P(\%)=\frac{\text { Peso médio final }- \text { Peso médio inicial }}{\text { Peso médio inicial }} \times 100 \\
& A V(\%)=\frac{\text { Peso médio final }- \text { Peso médio inicial }}{\text { Volume médio inicial }} \times 100
\end{aligned}
$$

Onde: Peso médio final - Peso médio inicial é considerado o volume d'água absorvida, tendo em vista convencionar a densidade da água igual a $1 \mathrm{~g} /$ $\mathrm{cm}^{3}$.

$I E(\%)=\frac{\text { Espessura média final }- \text { Espessura média inicial }}{\text { Espessura média inicial }} \times 100$

Também, com os dados obtidos do procedimento de pesagem e medição das peças na condição de equilíbrio higroscópico em câmara climatizada, e do peso seco após secagem a $103^{\circ} \mathrm{C}$ até massa constante, determinou-se, para todos os corpos de prova testados, a densidade aparente (Da) e a densidade básica (Db), ambas ao teor de umidade de equilíbrio, após condicionamento a $12 \%$ de umidade, conforme as Equações 4 e 5.

$$
\begin{aligned}
& D a=\frac{\text { Peso ao teor de umidade de equilíbrio }(\mathrm{g})}{\text { Volume ao teor de umidade de equilíbrio }\left(\mathrm{cm}^{3}\right)} \\
& D b=\frac{\text { Peso seco }(\mathrm{g})}{\text { Volume ao teor de umidade de equilíbrio }\left(\mathrm{cm}^{3}\right)}
\end{aligned}
$$

Os ensaios mecânicos foram executados em Máquina Universal de Ensaios, hidráulica, marca AMSLER com capacidade de 20 toneladas, pertencente ao Laboratório de Produtos Florestais da UFSM.

Da mesma forma que nos testes de absorção d'água e inchamento em espessura, foram determinadas as densidades básicas, aparente e teor de umidade de todos os corpos de prova testados à umidade de equilíbrio (TUe).

No teste de flexão estática, determinaram-se diretamente o módulo de ruptura (MOR) e o módulo de elasticidade (MOE) em corpos de prova de 350 $\mathrm{mm}$ de comprimento e $75 \mathrm{~mm}$ de largura, ambos em $\mathrm{kgf} / \mathrm{cm}^{2}$. A seguir, estes corpos de prova retornaram ao condicionamento e em outra ocasião, depois de atingido novo equilíbrio higroscópico, procedeu-se 
nos mesmos, ao teste de arrancamento de parafuso em que se determinou a carga necessária para tal, expressa em kgf.

Para avaliar a qualidade de colagem dos adesivos utilizados, determinaram-se os valores de ligação interna em corpos de prova de 50 x 50 $\mathrm{mm}$, por meio do teste de tração perpendicular à superfície da chapa, registrando-se os valores em kgf. Devido à característica destrutiva deste teste, retiraram-se das chapas amostras adjacentes aos locais de retirada dos mesmos, de iguais dimensões, para determinação do teor de umidade (TUe) e massa específica básica $(\mathrm{Db})$.

Para auxiliar na interpretação do comportamento dos painéis de cada tratamento, os dados obtidos dos ensaios físico-mecânicos descritos, foram correlacionados com as variáveis de fabricação das chapas: densidade aparente (Da), densidade básica $(\mathrm{Db})$, taxa de compressão real (TXR) e o teor de umidade nas condições avaliadas (TUe, TU2h, TU24h). A taxa de compressão real foi calculada devido à discrepância observada entre os valores de densidade básica dos corpos de prova testados, havendo, portanto, a necessidade de correção da taxa de compressão nominal prevista de 1,26 , para se proceder a análise de correlação entre as variáveis. Então, a partir da relação entre as densidades básicas de cada corpo de prova e da madeira utilizada, calculou-se para cada um a taxa de compressão real (TXR), obtendo-se os valores médios de 1,17 e 1,27, para as chapas confeccionadas com os adesivos à base de ureia $\mathrm{e}$ tanino, respectivamente.

\section{RESULTADOS E DISCUSSÃO}

\section{Absorção d'água e inchamento em espessura}

Os valores médios dos teores de umidade nas três condições de avaliação, densidade básica e aparente à umidade de equilíbrio de $12 \%$, absorção d'água em peso, absorção d'água em volume e inchamento em espessura após 2 e 24 horas de imersão, para os painéis à base de ureia e de taninoformaldeído, são apresentados na Tabela 1 .

Observa-se na Tabela 1 que os valores de densidade básica e densidade aparente das chapas com diferentes adesivos diferiram estatisticamente e, considerando que foram utilizadas partículas puras da mesma espécie e componentes da chapa de mesma proporção, essa diferença de aproximadamente $6 \%$, pode ser atribuída à maior viscosidade do adesivo à base de tanino em relação àquele à base de ureia, o que contribuiu para o aumento de massa pelo painel. De certa forma, esse comportamento das chapas, bem como as características conferidas ao adesivo à base de tanino, pode ter contribuído para o inchamento em espessura estatisticamente inferior, refletindo também nas menores médias apresentadas para as demais variáveis analisadas, porém sem diferença estatística.

Os valores de inchamento em espessura para ambos os tratamentos após imersão por 2 e 24 horas em água, foram inferiores a 6 e 15\%, respectivamente, que são os limites máximos permitidos pela norma DIN 68761 (3)-1971.

Em comparação com Iwakiri et al. (2004), em chapas confeccionadas com uréia-formaldeído,

TABELA 1: Valores médios de absorção d'água e inchamento em espessura após 2 e 24 horas de imersão dos painéis para os dois tratamentos, com resultado do teste de médias e respectivos coeficientes de variação.

TABLE 1: Mean values of water absorption and thickness swelling after 2 and 24 hours of immersion of particleboard for the two treatments.

\begin{tabular}{cccccccccccc}
\hline Trat. & TUe & TU2h & TU24h & Da & Db & AP2h & AP24h & AV2h & AV24h & IE2h & IE24h \\
\hline UF & $9,71 \mathrm{a}$ & $17,40 \mathrm{a}$ & $39,84 \mathrm{a}$ & $0,66 \mathrm{~b}$ & $0,59 \mathrm{~b}$ & $7,01 \mathrm{a}$ & $27,45 \mathrm{a}$ & $4,50 \mathrm{a}$ & $17,79 \mathrm{a}$ & $4,31 \mathrm{a}$ & $13,40 \mathrm{a}$ \\
TF & $9,54 \mathrm{a}$ & $16,61 \mathrm{a}$ & $37,22 \mathrm{a}$ & $0,70 \mathrm{a}$ & $0,63 \mathrm{a}$ & $6,45 \mathrm{a}$ & $25,25 \mathrm{a}$ & $4,22 \mathrm{a}$ & $17,23 \mathrm{a}$ & $3,01 \mathrm{~b}$ & $9,73 \mathrm{~b}$ \\
CV\% & 6,07 & 8,17 & 11,15 & 5,55 & 5,43 & 11,24 & 12,60 & 8,06 & 9,03 & 26,43 & 13,54 \\
\hline
\end{tabular}

Em que: $\mathrm{UF}=$ Ureia-formaldeído; $\mathrm{TF}=$ Tanino-formaldeído $\mathrm{CV}=$ Coeficiente de variação; $\mathrm{TUe}=$ Teor de umidade de equilíbrio a $20^{\circ} \mathrm{C}$ e $65 \% \mathrm{UR},(\%)$; TU2h $=$ Teor de umidade após 2 horas de imersão, (\%); TU24h $=$ Teor de umidade após 24 horas de imersão, (\%); $\mathrm{Da}=$ Densidade aparente ao teor de umidade de equilíbrio, $\left(\mathrm{g} / \mathrm{cm}^{3}\right) ; \mathrm{Db}=$ Densidade básica $\left(\mathrm{g} / \mathrm{cm}^{3}\right) ; \mathrm{AP} 2 \mathrm{~h}=$ Absorção d'água em peso após 2 horas de imersão, $(\%) ; \mathrm{AP} 24 \mathrm{~h}=$ Absorção d'água em peso após 24 horas de imersão, (\%); AV2h = Absorção d'água em volume após 2 horas de imersão, (\%); AV24h = Absorção d'água em volume após 24 horas de imersão, (\%); IE2h = Inchamento em espessura após 2 horas de imersão, (\%); IE24h = Inchamento em espessura após 24 horas de imersão, (\%). Médias seguidas pela mesma letra não diferem estatisticamente entre si pelo teste $\mathrm{T}$ ao nível de $5 \%$ de probabilidade de erro. 
que registraram um inchamento em espessura de 8,57 e $17,71 \%$, e de absorção d'água em peso de 15,81 e $42,91 \%$, após imersão em água por 2 e 24 horas, respectivamente, observa-se que os valores registrados em ambas as condições de avaliação para a madeira de canafístula, foram inferiores aos registrados pelos autores, que também observaram inchamentos em espessura superiores aos limites convencionados pela norma acima.

Já com relação aos dados de Dacosta et al. (2005a), nos quais encontraram valores de AP2h de $11,70 \%$ e AP24h de $58,93 \%$, IE2h de 3,71\% e IE24h de $21,49 \%$, tem-se que somente o IE2h se mostrou próximo, embora um pouco superior, enquanto as demais variáveis aqui encontradas foram bastante inferiores.

Em comparação com Iwakiri et al. (2000) em estudo com as madeiras de Eucalyptus maculata, E. grandis e E. tereticornis, as variáveis de absorção d'água e de inchamento foram bastante inferiores, visto que estes autores encontraram valores de AP2h entre 13,94 e $41,74 \%$, AP24h entre 37,37 e 58,80\%, IE2h entre 12,38 e 30,60 e IE24h entre 23,51 e $38,81 \%$.

Os valores de absorção d'água em peso e inchamento em espessura dos painéis à base de tanino-formaldeído após imersão em água por 2 horas ficaram próximos aos encontrados por Haselein et al. (2002), para Pinus elliottii, que foram de 11,87 e 4,72\%, respectivamente. Já os equivalentes em 24 horas foram bastante distintos, aumentando para 60,70 e $35,43 \%$.

Também, o valor de IE2h foi próximo, embora inferior ao encontrado por Hillig et al. (2004), trabalhando com o mesmo adesivo e as madeiras de Pinus elliottii, Eucalyptus grandis e Acacia mearnsii, em que obtiveram valores de absorção d'água em peso de 16,70 e $70,52 \%$, absorção d'água em volume de 11,13 e $47,05 \%$ e inchamento em espessura de 4,49 e $22,14 \%$ em 2 e 24 horas, respectivamente, portanto todas as demais bastante superiores aos aqui registrados.

Na Tabela 2 constam as correlações calculadas para os valores de absorção d'água em peso, absorção d'água em volume e inchamento em espessura com as propriedades de fabricação das chapas (Da, Db, TXR) e teores de umidade nas três condições de avaliação (TUe, TU2h, TU24h). Os valores em negrito na parte inferior da tabela correspondem aos painéis confeccionados com adesivo ureia-formaldeído e os da parte superior sem negrito referem-se aos painéis confeccionados com adesivo tanino-formaldeído.

TABELA 2: Correlações de Pearson entre as propriedades de absorção d'água e inchamento em espessura e as variáveis de fabricação das chapas.

TABLE 2: Pearson's correlations between the water absorption properties and thickness swelling and the variables of particleboard manufacture.

\begin{tabular}{|c|c|c|c|c|c|c|c|c|c|c|c|c|}
\hline & $\mathrm{Da}$ & $\mathrm{Db}$ & TXR & AP2h & AP24h & AV2h & AV24h & IE2h & IE24h & TUe & TU2h & TU24h \\
\hline $\mathrm{Da}$ & - & - & $0,99 * *$ & $0,94^{* *}$ & $-0,92 * *$ & $-0,89 * *$ & $-0,86^{* *}$ & $0,18^{\mathrm{ns}}$ & $-0,17^{\mathrm{ns}}$ & $-0,87 * *$ & $-0,94 * *$ & $0,93 * *$ \\
\hline $\mathrm{Db}$ & - & - & $0,99 * *$ & $-0,75^{*}$ & $-0,82 * *$ & $0,02^{\mathrm{ns}}$ & $0,37^{\mathrm{ns}}$ & $0,14^{\mathrm{ns}}$ & $0,31^{\mathrm{ns}}$ & $-0,95 * *$ & $-0,93 * *$ & $0,85^{* *}$ \\
\hline TXR & $0,99 * *$ & $0,99 * *$ & - & $-0,95 * *$ & $-0,93 * *$ & $-0,95^{* *}$ & $-0,86^{* *}$ & $0,18^{\mathrm{ns}}$ & $-0,19^{\text {ns }}$ & $-0,90 * *$ & $-0,95^{* *}$ & $0,94 * *$ \\
\hline $\mathrm{AP} 2 \mathrm{~h}$ & $-0,79 *$ & $-0,75^{*}$ & $-0,79 *$ & - & $0,99 * *$ & $0,99 * *$ & $0,97 * *$ & $0,03^{\text {ns }}$ & $0,41^{\mathrm{ns}}$ & $0,89 * *$ & $0,98 * *$ & $0,99 * *$ \\
\hline AP24h & $-0,80 * *$ & $-0,82 * *$ & $-0,81 * *$ & $0,63^{\text {ns }}$ & - & $0,99 * *$ & $0,99 * *$ & $0,11^{\mathrm{ns}}$ & $0,46^{\mathrm{ns}}$ & $0,87 * *$ & $0,96 * *$ & $0,99 * *$ \\
\hline $\mathrm{AV} 2 \mathrm{~h}$ & $-0,04^{\text {ns }}$ & $0,02^{\text {ns }}$ & $-0,04^{\text {ns }}$ & $0,64^{\mathrm{ns}}$ & $\mathbf{0 , 0 3 ^ { \text { ns } }}$ & - & $0,99 * *$ & $0,15^{\mathrm{ns}}$ & $0,50^{\mathrm{ns}}$ & $0,87 * *$ & $0,96 * *$ & $0,99 * *$ \\
\hline AV24h & $0, \mathbf{3 3}^{\text {ns }}$ & $0,37^{\text {ns }}$ & $0,36^{\text {ns }}$ & $-0,31^{\text {ns }}$ & $0,03^{\text {ns }}$ & $-0,05^{\text {ns }}$ & - & $0,24^{\mathrm{ns}}$ & $0,55^{\text {ns }}$ & $0,82 * *$ & $0,93 * *$ & $0,98 * *$ \\
\hline IE2h & $-0,04^{\text {ns }}$ & $0,14^{\mathrm{ns}}$ & $0,13^{\text {ns }}$ & $-0,19^{\text {ns }}$ & $0,16^{\mathrm{ns}}$ & $-0,13^{\text {ns }}$ & $0,65^{\mathrm{ns}}$ & - & $0,67 *$ & $0,20^{\mathrm{ns}}$ & $-0,06^{\mathrm{ns}}$ & $0,06^{\mathrm{ns}}$ \\
\hline IE24h & $0,33^{\text {ns }}$ & $0,31^{\mathrm{ns}}$ & $0,34^{\text {ns }}$ & $-0,74 *$ & $-0,04^{\mathrm{ns}}$ & $-0,73 *$ & $0,53^{\text {ns }}$ & $0,58^{\text {ns }}$ & - & $0,20^{\text {ns }}$ & $0,33^{\text {ns }}$ & $0,42^{\mathrm{ns}}$ \\
\hline TUe & $-0,93 * *$ & $0,95 * *$ & $-0,93 * *$ & $0,59^{\text {ns }}$ & $0,87 * *$ & $-0,18^{\text {ns }}$ & $-0,31^{\text {ns }}$ & $0,02^{\text {ns }}$ & $-0,08^{\text {ns }}$ & - & $0,96 * *$ & $0,91 * *$ \\
\hline TU2h & $-0,94 * *$ & $-0,93 * *$ & $-0,95 * *$ & $0,92 * *$ & $0,82 * *$ & $0,3^{\text {ns }}$ & $-0,35^{\text {ns }}$ & $-0,11^{n s}$ & $-0,51^{\mathrm{ns}}$ & $0,85 * *$ & - & $0,98 * *$ \\
\hline TU24h & $-0,83 * *$ & $-0,85^{* *}$ & $-0,85 * *$ & $0,64^{\mathrm{ns}}$ & $0,98 * *$ & $0,003^{\text {ns }}$ & $-0,02^{\text {ns }}$ & $0,14^{\mathrm{ns}}$ & $-0,04^{\mathrm{ns}}$ & $0,90 * *$ & $0,84 * *$ & - \\
\hline
\end{tabular}

Em que: $\mathrm{Da}=$ Densidade aparente ao teor de umidade de equilíbrio $\left(\mathrm{g} / \mathrm{cm}^{3}\right) ; \mathrm{Db}=$ Densidade básica $\left(\mathrm{g} / \mathrm{cm}^{3}\right) ; \mathrm{TXR}=$ Taxa de compressão real; AP2h = Absorção em peso após 2 horas de imersão (\%); AP24h = Absorção em peso após 24 horas de imersão, (\%); AV2h = Absorção em volume após 2 horas de imersão, (\%); AV24h = Absorção em volume após 24 horas de imersão, (\%); IE2h = Inchamento em espessura após 2 horas de imersão; IE24h = Inchamento em espessura após 24 horas de imersão; TUe = Teor de umidade de equilíbrio, $(\%)$; TU2h $=$ Teor de umidade após 2 horas de imersão, (\%); TU24h = Teor de umidade após 24 horas de imersão, $(\%) ; * *$ = Correlação significativa ao nível de $1 \%$ de probabilidade, pelo teste $\mathrm{T} ; *=$ Correlação significativa ao nível de $5 \%$ de probabilidade pelo teste $\mathrm{T}$; ${ }^{\mathrm{ns}}=$ Correlação não significativa ao nível de 1 e $5 \%$ de probabilidade de erro, pelo teste T. 
De uma maneira geral, os painéis confeccionados com tanino apresentaram um maior número de correlações e, também, de significâncias em comparação com os fabricados a partir do adesivo à base de ureia, o que demonstra uma maior homogeneidade do material fabricado, ao longo das avaliações.

A análise do quadro de correlação mostrou um comportamento similar para os dois tratamentos no que diz respeito à influência das variáveis de confecção. Para as chapas à base de ureia, as variáveis de fabricação das chapas e os teores de umidade nas três condições correlacionaram-se inversamente entre si e com a absorção em peso em 2 e 24 horas (AP2h, AP24h) sendo que nos demais casos não houve correlação. Já para o tanino as correlações só não foram significativas para o inchamento em espessura em 2 e 24 horas (IE2h e IE24h).

Observa-se a alta correlação entre AP2h e $\mathrm{AP} 24 \mathrm{~h}$ dos painéis à base de tanino com AV2h e AV24h, o que denota a possibilidade de estimativa das últimas a partir das primeiras, levando em consideração a facilidade e a maior precisão das medidas de peso em relação à tomada de medidas para cálculo do volume. Na Figura 2 consta a equação para estimativa da AV24h a partir dos dados de AP24h.

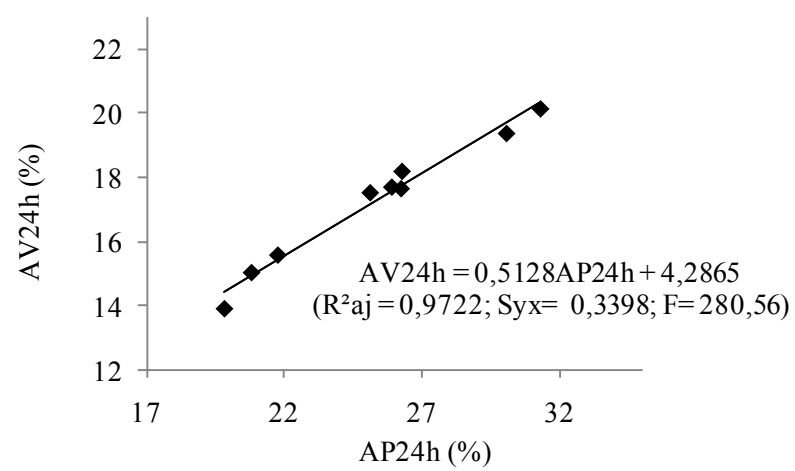

FIGURA 2: Estimativa da percentagem de absorção d'água em volume após imersão por 24 horas (AV24h), para os painéis aglomerados à base de taninoformaldeído, a partir dos valores de absorção d'água em peso após imersão por 24 horas (AP24h).

FIGURE 2: Water absorption after 24 hours (AV24h) of the particleboard glued with tannin-formaldehyde as a function of water absorption after 24 hours (AP24h).
Com menor grau de correlação pode-se estimar os valores de IE24h dos painéis à base de ureia a partir AP2h $(R=-0,74)$, conforme Figura 3 .

\section{Propriedades mecânicas}

$\mathrm{Na}$ Tabela 3 constam os valores médios obtidos para o MOE e MOR a partir do teste de flexão estática, ligação interna e resistência ao arrancamento de parafuso, juntamente com o teste de média e respectivos coeficientes de variação.

A Tabela 3 mostra que todas as propriedades mecânicas dos painéis confeccionados com tanino foram superiores às obtidas pelos confeccionados com adesivo à base de ureia, e que suas médias diferiram-se estatisticamente entre si pelo teste $\mathrm{T}$, com exceção do MOR. A qualidade das propriedades mecânicas dos painéis foi definida em consulta às normas DIN 68761 (1)-1961, e ANSI A208.11987, a primeira cita valor mínimo de ligação interna de $3,5 \mathrm{kgf} / \mathrm{cm}^{2}$, a segunda estipula valores mínimos de MOE e MOR de 17.590 e $112 \mathrm{kgf} / \mathrm{cm}^{2}$, respectivamente e resistência ao arrancamento de parafuso de $102 \mathrm{kgf}$.

Portanto, com base nestes valores, os painéis de canafístula fabricados com o adesivo tanino, com exceção da resistência ao arrancamento de parafuso, obtiveram valores de resistência mecânica acima do mínimo estipulado pelas normas, já o MOR dos

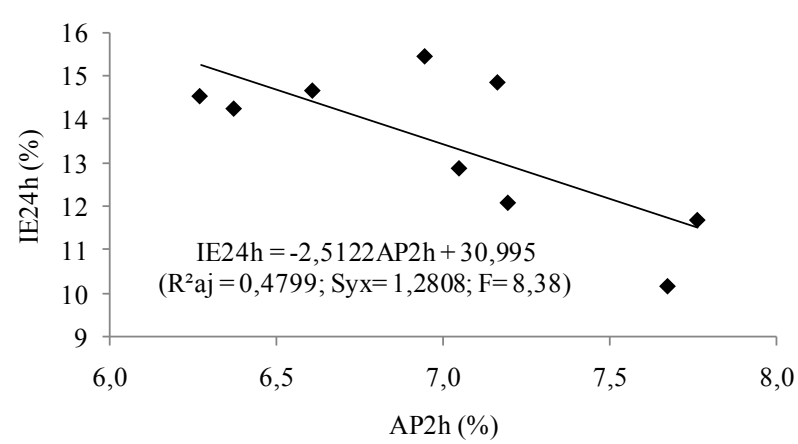

FIGURA 3: Estimativa da percentagem de inchamento em espessura após 24 horas de imersão (IE24h) para os painéis aglomerados à base de ureiaformaldeído a partir dos valores de absorção d'água em peso após imersão por 2 horas (AP2h).

FIGURE 3: Thickness swelling estimate after 24 hours of water immersion (IE24h) of the particleboard with ureaformaldehyde as a function of water absorption after 2 hours (AP2h). 
TABELA 3: Valores médios relativos aos testes mecânicos nas chapas aglomeradas com teste de média e respectivos coeficientes de variação.

TABLE 3: Average values of mechanical properties of the particleboard with the results of the test as well as coefficient of variation.

\begin{tabular}{l|c|c|c|c}
\hline TRATAMENTO & $\begin{array}{c}\mathrm{MOE} \\
\left(\mathrm{kgf} / \mathrm{cm}^{2}\right)\end{array}$ & $\begin{array}{c}\text { MOR } \\
\left(\mathrm{kgf} / \mathrm{cm}^{2}\right)\end{array}$ & $\begin{array}{c}\text { Ligação Interna } \\
\left(\mathrm{kgf} / \mathrm{cm}^{2}\right)\end{array}$ & $\begin{array}{c}\text { RAP } \\
(\mathrm{kgf})\end{array}$ \\
\hline Ureia-formaldeído & $15802,88 \mathrm{~b}$ & $171,34 \mathrm{a}$ & $1,94 \mathrm{~b}$ & $56,88 \mathrm{~b}$ \\
Tanino-formaldeído & $21322,49 \mathrm{a}$ & $207,05 \mathrm{a}$ & $5,70 \mathrm{a}$ & $91,88 \mathrm{a}$ \\
Coeficiente de variação\% & 25,24 & 25,58 & 47,79 & 20,91 \\
\hline
\end{tabular}

Em que: $\mathrm{MOE}=$ Módulo de elasticidade; $\mathrm{MOR}=$ Módulo de ruptura; $\mathrm{RAP}=$ Resistência ao arrancamento de parafuso . Médias seguidas pela mesma letra não diferem estatisticamente entre si pelo teste $\mathrm{T}$ ao nível de $5 \%$ de probabilidade.

painéis à base de ureia foi a única propriedade que alcançou os valores mínimos estipulados.

O maior coeficiente de variação para a ligação interna é devido, em alguns painéis, à linha de rompimento nos corpos de prova não ter ocorrido no mesmo ponto, o que gerou maior discrepância dos valores de carga obtidos no teste, dentro dos tratamentos.

Iwakiri et al. (2004), encontraram nos painéis confeccionados com Grevillea robusta e ureia-formaldeído valores de MOE de $14.630 \mathrm{kgf} /$ $\mathrm{cm}^{2}$ e MOR de $103 \mathrm{kgf} / \mathrm{cm}^{2}$, inferiores ao obtido neste estudo, no entanto a ligação interna encontrada de $7,21 \mathrm{kgf} / \mathrm{cm}^{2}$ foi bastante superior. Porém ao comparar a carga obtida para esta última propriedade com o encontrado por Iwakiri et al. (2005), de $1,9 \mathrm{kgf} / \mathrm{cm}^{2} \mathrm{com}$ o mesmo adesivo e a madeira de Pinus spp., observa-se que o valor de resistência foi praticamente o mesmo. Esses autores, no mesmo estudo, concluíram que esta propriedade, bem como o módulo de ruptura e o módulo de elasticidade, obtidos, foram dependentes da densidade do painel, independente da quantidade de resina utilizada.

Carga similar foi encontrada para o $\mathrm{MOE}$ em comparação com o trabalho de Brito et al. (2006), com a madeira de Pinus elliottii e ureia-formaldeído, que obtiveram valores de MOE de 15.370,93 kgf/ $\mathrm{cm}^{2}$, já o MOR pelos mesmos autores foi inferior e da ordem de $111,80 \mathrm{kgf} / \mathrm{cm}^{2}$ e a L.I de $4,12 \mathrm{kgf} / \mathrm{cm}^{2}$, mostrou-se bastante superior.

Numa comparação com os valores de carga obtidos por Dacosta et al. (2005b), para a LI de 1,65 $\mathrm{kgf} / \mathrm{cm}^{2}$, MOR de $96,11 \mathrm{kgf} / \mathrm{cm}^{2}$ e MOE 11.826 $\mathrm{kgf} / \mathrm{cm}^{2}$, os painéis com a madeira de canafístula apresentaram valores de resistência superiores. Porém, somente a RAP de $75 \mathrm{kgf}$ registrada por estes autores foi superior ao aqui registrado.

Já Gonçalves et al. (2003), obtiveram apenas MOR inferior, da ordem de 114,8 kgf/ $/ \mathrm{cm}^{2}$, já o encontrado para o MOE de $19.611 \mathrm{kgf} / \mathrm{cm}^{2}$ e LI de $6,98 \mathrm{kgf} / \mathrm{cm}^{2}$, superaram o aqui registrado. Por fim para os painéis confeccionados com o adesivo ureia-formaldeído, observa-se que somente a carga obtida para o MOE está dentro da faixa de resistência registrada por Iwakiri et al. (2000), que encontraram, para os diversos tratamentos empregados, valores de LI entre 7,74 e 13,63 kgf $/ \mathrm{cm}^{2}$, MOE entre 12.178 e $23.055 \mathrm{kgf} / \mathrm{cm}^{2}$ e MOR entre 74,13 e 163,36 kgf/ $\mathrm{cm}^{2}$.

Com relação aos painéis à base de taninoformaldeído Haselein et al. (2002) encontraram valores para o MOE de $19.006 \mathrm{kgf} / \mathrm{cm}^{2}$, MOR de $106,02 \mathrm{kgf} / \mathrm{cm}^{2}$, ligação interna de $1,85 \mathrm{kgf} / \mathrm{cm}^{2}$ e arrancamento de parafuso de 155,35 kgf, sendo que somente a última propriedade superou o valor obtido na presente pesquisa para a madeira de canafístula.

Com exceção do MOE, os valores aqui obtidos também foram superiores aos registrados por Vital et al. (2004), em chapas confeccionadas com o adesivo tanino obtido de Eucalyptus pellita, em que encontraram valores de L.I de $3,3 \mathrm{kgf} / \mathrm{cm}^{2}$, MOR de $181,9 \mathrm{kgf} / \mathrm{cm}^{2}$ e um MOE de $26.743 \mathrm{kgf} / \mathrm{cm}^{2}$.

A Tabela 4 apresenta uma análise das correlações existentes entre as variáveis de fabricação das chapas e as propriedades mecânicas obtidas dos corpos de prova a partir dos dois tipos de adesivos utilizados. Os valores em negrito na parte inferior da tabela correspondem aos painéis confeccionados com adesivo ureia-formaldeído e os da parte superior sem negrito referem-se aos painéis confeccionados com adesivo tanino-formaldeído.

Observa-se a alta correlação negativa entre as variáveis de produção das chapas $(\mathrm{Da}, \mathrm{Db}$, TXR) e o teor de umidade na condição do teste (Tue), sendo esta correlação ligeiramente superior para o painel à base de ureia. Também, para os dois tratamentos, não se observou influência dos teores de umidade na resistência mecânica oferecida pelos corpos de prova. 
TABELA 4: Correlações de Pearson entre as propriedades de resistência mecânica e as variáveis de fabricação das chapas.

TABLE 4: Pearson's correlations between the mechanical properties and the particleboard manufacture parameters.

\begin{tabular}{|c|c|c|c|c|c|c|c|c|}
\hline & $\mathrm{Da}$ & $\mathrm{Db}$ & RAP & LI & MOE & MOR & TXR & TUe \\
\hline $\mathrm{Da}$ & - & - & $0,64^{\mathrm{ns}}$ & $0,08^{\mathrm{ns}}$ & $0,91 * *$ & $0,38^{\mathrm{ns}}$ & $0,99 * *$ & $-0,87 * *$ \\
\hline $\mathrm{Db}$ & - & - & $0,64^{\mathrm{ns}}$ & $0,07^{\mathrm{ns}}$ & $0,91 * *$ & $0,40^{\text {ns }}$ & $0,99 * *$ & $-0,88^{* *}$ \\
\hline RAP & $0,79 *$ & $0,89 * *$ & - & $0,11^{\text {ns }}$ & $0,83^{*}$ & $0,56^{\mathrm{ns}}$ & $0,66^{\mathrm{ns}}$ & $-0,63^{\mathrm{ns}}$ \\
\hline LI & $\mathbf{0 , 2 0 ^ { \mathrm { ns } }}$ & $\mathbf{0 , 3 3} 3^{\text {ns }}$ & $-0,28^{\mathrm{ns}}$ & - & $0,70^{\mathrm{ns}}$ & $0,34^{\mathrm{ns}}$ & $-0,08^{\mathrm{ns}}$ & $-0,17^{\mathrm{ns}}$ \\
\hline MOE & $0,31^{\text {ns }}$ & $0,55^{\mathrm{ns}}$ & $0,69^{\text {ns }}$ & $-0,20^{\mathrm{ns}}$ & - & $0,66^{\mathrm{ns}}$ & $0,92^{* *}$ & $-0,48^{\mathrm{ns}}$ \\
\hline MOR & $0,59^{\text {ns }}$ & $0,69^{\text {ns }}$ & $0,64^{\mathrm{ns}}$ & $-0,01^{n s}$ & $0,80^{*}$ & - & $0,40^{\text {ns }}$ & $-0,09^{\mathrm{ns}}$ \\
\hline TXR & $1,00 * *$ & $0,99 * *$ & $0,87 * *$ & $0,31^{\mathrm{ns}}$ & $\mathbf{0 , 5 0} \mathbf{n s}^{\mathrm{ns}}$ & $0,67^{\text {ns }}$ & - & $-0,90^{* *}$ \\
\hline TUe & $-0,93 * *$ & $-0,95 * *$ & $-0,23^{\mathrm{ns}}$ & $-0,21^{\mathrm{ns}}$ & $-0,53^{\text {ns }}$ & $-0,05^{n s}$ & $-0,93^{* * *}$ & - \\
\hline
\end{tabular}

Em que: $\mathrm{Da}=$ Densidade aparente ao teor de umidade de equilíbrio, $\left(\mathrm{g} / \mathrm{cm}^{3}\right) ; \mathrm{Db}=$ Densidade básica, $\left(\mathrm{g} / \mathrm{cm}^{3}\right) ; \mathrm{RAP}=$ Resistência ao arrancamento de parafuso (kgf); LI= Ligação interna, $\left(\mathrm{kgf} / \mathrm{cm}^{2}\right) ; \mathrm{MOE}=$ Módulo de elasticidade, $(\mathrm{kgf} /$ $\left.\mathrm{cm}^{2}\right) ; \mathrm{MOR}=$ Módulo de ruptura, $\left(\mathrm{kgf} / \mathrm{cm}^{2}\right) ; \mathrm{TXR}=$ Taxa de compressão real; TUe = Teor de umidade de equilíbrio, (\%); ** = Correlação significativa ao nível de $1 \%$ de probabilidade, pelo teste $\mathrm{T} ; *$ = Correlação significativa ao nível de $5 \%$ de probabilidade pelo teste $\mathrm{T}$; ${ }^{\text {ns }}=$ Correlação não significativa ao nível de 1 e $5 \%$ de probabilidade de erro, pelo teste $\mathrm{T}$.

Para a resistência ao arrancamento de parafuso (RAP), os painéis à base de ureia apresentaram correlação exclusivamente com as variáveis de fabricação das chapas, em maior grau com a densidade básica $(\mathrm{R}=0,89)$, conforme Figura 4 , seguida da taxa de compressão real $(\mathrm{R}=0,87)$ e da densidade aparente $(\mathrm{R}=0,79)$, enquanto que no tratamento à base de tanino esta propriedade correlacionou-se apenas com o módulo de elasticidade $(\mathrm{R}=0,83)$.

A propriedade de ligação interna não apresentou correlação com nenhuma das propriedades mecânicas e de fabricação das chapas para os dois tipos de adesivo, não sendo, portanto, possível estimar seus valores a partir de outra propriedade.

Com relação à análise dos resultados para o teste de flexão estática dos painéis à base de tanino, a Tabela 4 mostra que o MOE apresentou correlação significativa ao nível de 5\% com as propriedades de fabricação das chapas: densidade aparente e básica $(\mathrm{R}=0,91)$ e taxa de compressão real $(\mathrm{R}=$ 0,92), conforme Figura 5, e ao nível de 1\% com a resistência mecânica ao arrancamento de parafuso

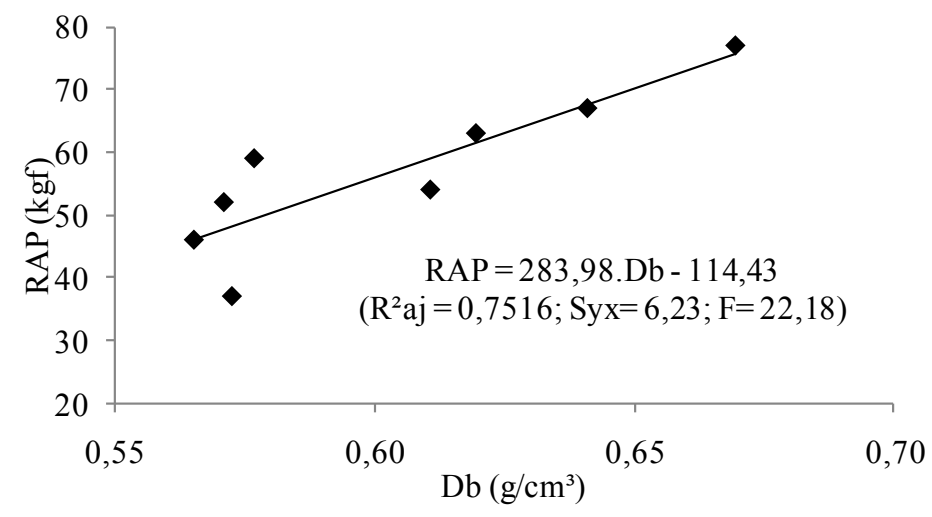

FIGURA 4: Estimativa dos valores de resistência ao arrancamento de parafuso (RAP) dos painéis à base de ureia-formaldeído a partir da densidade básica da chapa (Db).

FIGURE 4: Estimative of values of screw withdrawal (RAP) of the particleboard with urea-formaldehyde as a function of the basic density of the board $(\mathrm{Db})$. 
$(\mathrm{R}=0,83)$, não sendo, porém, observada correlação significativa entre o MOR obtido dos mesmos painéis com alguma das variáveis analisadas.

Quanto aos painéis à base de ureia observase que, embora em menor grau, podem-se estimar os valores de MOE a partir dos dados de MOR $(\mathrm{R}=0,80)$, sendo que este último não apresentou correlação com nenhuma outra variável. A Figura 6 mostra o gráfico que relaciona o MOE em função do MOR com os valores observados e ajustados por regressão linear.

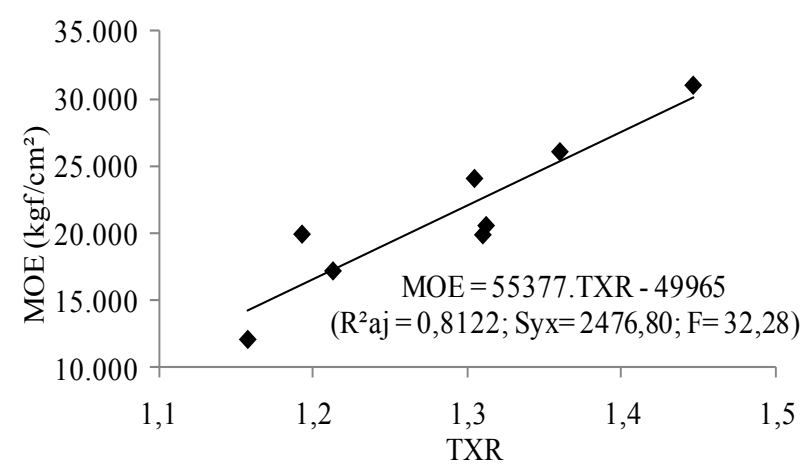

FIGURA 5: Estimativa do módulo de elasticidade (MOE) dos painéis colados com tanino-formaldeído a partir da taxa de compressão real (TXR).

FIGURE 5: Modulus of elasticity (MOE) of the particleboard glued with tanninformaldehyde as a function of compression rate (TXR).

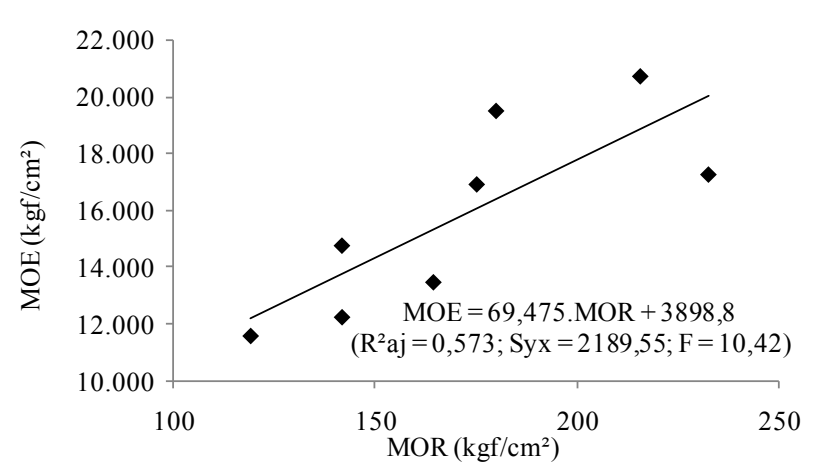

FIGURA 6: Estimativa do módulo de elasticidade (MOE) dos painéis à base de ureiaformaldeído a partir do módulo de ruptura (MOR).

FIGURE 6: Estimative of elastic modulus (MOE) of the particleboard with ureaformaldehyde as a function of modulus of rupture (MOR).

\section{CONCLUSÕES}

Ambos os adesivos permitiram confeccionar painéis com propriedades físicas satisfatórias, visto que os resultados de inchamento em espessura ficaram abaixo do limite pelos padrões de comercialização DIN 68761 (3)-1971, e as demais propriedades com valores bastante inferiores aos encontrados por outros autores com painéis de características similares.

Os painéis confeccionados com taninoformaldeído apresentaram resistência mecânica estatisticamente superior e, com exceção da resistência ao arrancamento de parafusos, todas as variáveis testadas superaram o mínimo estabelecido pelas normas DIN 68761 (1)-1971 e ANSI A208.11987. Já naqueles confeccionados com ureiaformaldeído apenas o módulo de ruptura foi superior ao mínimo admitido pelos padrões utilizados.

As partículas da madeira de Peltophorum dubium quando unidas com adesivo à base de tanino-formaldeído permitiram a fabricação de chapas com características tecnológicas superiores às confeccionadas com adesivo ureia-formaldeído, fazendo desta espécie uma fonte alternativa promissora na utilização para produção de painéis de madeira reconstituída.

Recomenda-se a condução de estudos com partículas desta mesma espécie misturada a outras, bem como sua utilização em maiores dimensões e proporção de adesivo, de maneira a se obter cargas maiores para a ligação interna, uma vez que ficaram bem abaixo do encontrado em outros trabalhos, denunciando baixa qualidade da colagem.

\section{REFERÊNCIAS BIBLIOGRÁFICAS}

AMERICAN NATIONAL STANDARDS INSTITUTE. Mat formed wood particleboard. ANSI-A-208.1, 1987.

AMERICAN SOCIETY FOR TESTING AND MATERIALS. Standard methods of evaluating properties of wood-base fiber and particle materials. ASTM D1037 - 93, 1992.

AZAMBUJA, M. A. et al. Análise da viabilidade de utilização de resíduos de maçaranduba na produção de painéis de madeira aglomerada, 2006. citation on computers documents. Disponível em: <(http://www.ppgec.feis.unesp.br/ producao2006/2.pdf) > Acesso em: 23 de janeiro de 2009.

BRITO, E. O. et al. Chapas de madeira aglomerada 
utilizando partículas oriundas de madeira maciça e de maravalhas. Scientia Forestalis, Piracicaba, v. 1, n. 72, p. 17-21, dez. 2006.

BRITO, E. O. et al. Propriedades de chapas produzidas com resíduo do fruto de coco e partículas de pinus. Floresta e Ambiente, Seropédica, v. 11, n. 2, p. 1-6, ago./dez. 2004.

CARASCHI, J. C. et al. Avaliação de painéis produzidos a partir de resíduos sólidos para aplicação na arquitetura. Polímeros: ciência e tecnologia, São Carlos, v. 19, n. 1, p. 47-53, mar. 2009.

DACOSTA, L. P. E. et al. Propriedades físicas de chapas de partículas aglomeradas fabricadas com resíduos de processamento mecânico da madeira de Pinus elliotii Engelm. Ciência Florestal, Santa Maria, v. 15, n. 4, p. 421-429, out./dez. 2005 a.

DACOSTA, L. P. E. et al. Qualidade das chapas de partículas aglomeradas fabricadas com resíduos do processamento mecânico da madeira de Pinus elliotii Engelm. Ciência Florestal, Santa Maria, v. 15, n. 3, p. 311-322, jul./set. 2005b.

DEUTSCHES INSTITUT FÜR NORMUNGE. Testing of wood chipboards-determination of variation in thickness due to moisture. DIN 68761, (1)-1961, (3)-1971.

GONÇALVES, C. de A. et al. Produção de chapas de madeira aglomerada com adesivo uréiaformaldeído modificado com tanino de Mimosa caesalpiniaefolia bentham (sabiá). Floresta e Ambiente, Seropédica, v. 10, n. 1, p. 18-26, jan./ jul. 2003.

HASELEIN, C. R. et al. Resistência mecânica e à umidade de painéis aglomerados com partículas de madeira de diferentes dimensões. Ciência Florestal, Santa Maria, v. 12, n. 2, p. 127 - 134, dez. 2002.

HILLIG, E. et al. Estabilidade dimensional de chapas aglomeradas estruturais (flakeboards) fabricadas com madeiras de Pinus, eucalipto e acácia-negra. Scientia Forestalis, Piracicaba, n. 65, p. $80-94$, jun. 2004.

HILLIG, E. etal.Qualidade de chapas aglomeradas estruturais, fabricadas com madeiras de pinus, eucalipto e acácia-negra, puras ou misturadas, coladas com tanino-formaldeído. 2000. 96 f. Dissertação (Mestrado em Engenharia Florestal)Universidade Federal de Santa Maria, Santa Maria, 2000.

INOUE, M. T. et al. Projeto madeira do Paraná. Curitiba: Fundação de Pesquisas Florestais do
Paraná, 1984. $260 \mathrm{p}$.

IPT - Instituto de Pesquisas Tecnológicas do Estado de São Paulo. Fichas de características das madeiras brasileiras. São Paulo: IPT, 1978. s.p.

IWAKIRI, S. et al. Produção de chapas de madeira aglomerada de cinco espécies de pinus tropicais. Floresta e Ambiente, Seropédica, v. 8, n.1, p.137 142, jan./dez. 2001.

IWAKIRI, S. et al. Produção de painéis aglomerados de alta densificação com uso de resina melaninauréia-formaldeído. Cerne, Lavras, v. 11, n. 4, p. 323-328, out./dez. 2005.

IWAKIRI, S. et al. Produção de painéis de madeira aglomerada de Grevillea robusta A. Cunn. Ex R. Br. Revista Árvore, Viçosa, v. 28, n. 6, p. 883-887, nov./dez. 2004.

IWAKIRI, S. et al. Resíduos de serrarias na produção de painéis de madeira aglomerada de eucalipto. Scientia Agraria, Curitiba, v. 1, n. 1, p. 23-28, jan/dez. 2000.

MACIEL, A. da, S. et al. Chapas de madeira aglomerada produzidas com partículas de Eucalyptus grandis W. Hill ex Maiden, poliestireno (PS) e polietileno tereftalato (PET). Cerne, Lavras, v. 10, n. 1, p. 53-66, jan./jun. 2004.

NAHUZ, M. A. R. Uso racional de produtos florestais, 2001. citation on computers documents. Disponível em: <(http:// www.remade.com.br/br/revistadamadeira materia.php? num $=53 \&$ subject $=$ Uso $\% 20$ Racional\&title $=$ Uso $\% 20$ Racional $\% 20 \mathrm{de} \% 20$ produtos $\% 20$ Florestais) $>$ Acesso em: 23 de janeiro de 2009.

NASCIMENTO, M. F., LAHR, F. A. R. Tecnologia de CPH- Chapas de Partículas Homogêneas Associada a sustentabilidade da região nordeste do Brasil, 2003. citation on computers documents. Disponível em: <(http://www.esac.pt/cernas/cfn5/ docs/T4-29.pdf) $>$ Acesso em: 25 de janeiro de 2009.

NUNES, W. H. et al. Produção de chapas de partículas do estipe de Euterpe edulis Martius (palmiteiro). Floresta e Ambiente, Seropédica, v. 6, n. 1, p. 95-105, jan./dez. 1999.

TEIXEIRA, M. G., CESÁR, S. F. Produção de compósito com resíduo de madeira no contexto da ecologia industrial, 2006. citation on computers documents. Disponível em: $\quad<$ (http://portal.faculdadedacidade.edu.br/ index 2.php?option $=$ com_docman\&task $=$ doc view\&gid=7\&Itemid=290) $>$ Acesso em: 23 de janeiro de 2009. 
TOSTES, A. de, S. et al. Colagem de chapas de madeira aglomerada com adesivo uréia-formaldeído (UF) modificado com tanino da casca de Eucalyptus pellita F. Muell. Floresta e Ambiente, Seropédica, v. 11, n.2, p. 14 - 19, ago./dez. 2004.

VIDAURRE, G. P. et al. Produção de chapas de partículas de madeira de duas espécies nativas da mata atlântica e suas combinações. Ciência Florestal, Santa Maria, v. 14, n. 1, p. 235 - 242, jun. 2004.
VITAL, B. R. et al. Adesivos à base de taninos das cascas de duas espécies de eucalipto para produção de chapas de flocos. Revista Árvore, Viçosa, v. 28, n. 4, p. 571-582, jul./ago. 2004.

VIVIAN, M. A. et al. Características tecnológicas da madeira de canafístula (Peltophorum dubium (Spreng.) Taub.) e seu comportamento na secagem ao ar livre. In: JORNADA ACADÊMICA INTEGRADA, 23., 2008, Santa Maria. Anais... Santa Maria: PRPGP-UFSM, 2008, 8 p. 